\title{
Bianisotropic route to the realization and matching of backward-wave metamaterial slabs
}

\author{
S. A. Tretyakov, ${ }^{1}$ C. R. Simovski, ${ }^{1,2}$ M. Hudlička ${ }^{1,3}$ \\ ${ }^{1}$ Radio Laboratory/SMARAD, Helsinki University of Technology, P.O. Box 3000, FI-02015 TKK, Finland \\ ${ }^{2}$ Physics Department, State University of Information Technologies, \\ Mechanics and Optics, Sablinskaya 14, 197101, St. Petersburg, Russia \\ ${ }^{3}$ Faculty of Electrical Engineering, Czech Technical University, Technická 2, 16627, Prague 6, Czech Republic
}

(Dated: August 1, 2020)

\begin{abstract}
A concept of backward-wave bianisotropic composite medium matched to free space is suggested. It is based on the use of a uniaxial bianisotropic structure embedded into a matrix with negative effective permittivity. Since bianisotropy is easier to achieve in the optical range than artificial magnetism, this concept is prospective for optical backward-wave metamaterials. As an example of possible realizations, a microwave $\Omega$-composite combined with a wire lattice is analytically studied.
\end{abstract}

PACS numbers: 78.20.Ci, 42.70.Qs, 42.25.Gy, 73.20.Mf, 78.67.Bf

Design and studies of materials with negative electromagnetic parameters supporting backward waves is currently a very active field of research. The concept of backward waves is not new: It goes back to the beginning of the 19th century and is connected to the names of Lamb, Schuster, and Pocklington. In the middle of the 20th century, this concept was extended to waves in homogeneous materials and negative refraction effect was theoretically predicted (Mandelshtam, Sivukhin, Silin, Veselago). Detailed reviews on the current status and on the history of this research field can be found in e.g. [1-7].

The main application for backward-wave materials is in sub-wavelength imaging devices ("perfect lens" [8][10]), but a full range of other possibilities is expected, especially for optical frequencies, if low-loss and matched slabs of backward-wave materials are realized $[6,11]$. The well-known design approach for the microwave range is based on the use of arrays of long thin metal conductors and split rings [12]. This approach has been extended to terahertz [13] and even infrared frequency range [14]. Realization of negative permeability with the use of split rings becomes very problematic at optical frequencies, and some alternative approaches have been proposed [18]-[21]. Considerable progress has been reported along this route, but the samples realized so far suffer from high losses and poor matching with free space [13, 14]. There exist possibilities to realize backward waves also in anisotropic media $[15,16]$ and in anisotropic waveguides [17], which do not necessarily require magnetic properties of materials, but are restricted to strongly anisotropic structures. In addition, difficulties to realize backwardwave samples matched to free space inhibit potential applications both in the microwave and in the optical regions.

Majority of researchers focus on the design of magnetodielectrics, where the backward-wave regime is realized when both the permittivity $\epsilon$ and permeability $\mu$ have negative real parts. Following to paper [22], the bianisotropy is usually considered as a factor that one should avoid in the design of backward-wave materials, and effort is often concentrated on the design of symmetrical variants of split rings which minimizes magnetoelectric coupling [23]. However, backward waves can exist in more general linear media, namely in bianisotropic media (e.g., in chiral media [24]-[26]). It was recently demonstrated that in chiral media it is possible to improve the characteristics of backward-wave regime [26, 27]. Moreover, as we will show in this paper, there is a possibility to benefit from more design freedom offered by additional material parameters. Here we will show that it is possible to design a bianisotropic material in such a way that it supports linearly-polarized backward waves and a slab made of this material is perfectly matched to free space for the normal direction of propagation.

The material can be realized as a composite with $\Omega$-shaped metal inclusions, and can be called omegamedium [28]. Since reliable analytical models of $\Omega$ particles and $\Omega$-media were developed and checked numerically and experimentally for the microwave range [29-31], we use these models (valid below $70-100 \mathrm{GHz}$ ) for a demonstration of the concept having in mind an optical realization as one of targets.

Reciprocal uniaxial $\Omega$-media obey the following constitutive relations [30]:

$\mathbf{D}=\overline{\bar{\epsilon}} \cdot \mathbf{E}+j \sqrt{\varepsilon_{0} \mu_{0}} K \overline{\bar{J}} \cdot \mathbf{H}, \quad \mathbf{B}=\overline{\bar{\mu}} \cdot \mathbf{H}+j \sqrt{\varepsilon_{0} \mu_{0}} K \overline{\bar{J}} \cdot \mathbf{E}$

Denoting the unit vector along the optical axis as $\mathbf{z}_{0}$ we can write the permittivity and permeability dyadics in the form:

$$
\overline{\bar{\epsilon}}=\varepsilon_{0}\left(\varepsilon_{t} \overline{\bar{I}}_{t}+\varepsilon_{z} \mathbf{z}_{0} \mathbf{z}_{0}\right), \quad \overline{\bar{\mu}}=\mu_{0}\left(\mu_{t} \overline{\bar{I}}_{t}+\mu_{z} \mathbf{z}_{0} \mathbf{z}_{0}\right),
$$

where $\overline{\bar{I}}_{t}$ is the two-dimension unit dyadic defined in the plane orthogonal to $\mathbf{z}_{0}: \overline{\bar{I}}_{t}=\mathbf{x}_{0} \mathbf{x}_{0}+\mathbf{y}_{0} \mathbf{y}_{0}$. The magnetoelectric dyadic is antisymmetric: $\overline{\bar{J}}=\mathbf{z}_{0} \times \overline{\bar{I}}_{t}$. Complex dimensionless parameter $K$ measures the magnetoelectric coupling effect. Eigenwaves in such media are lin- 
early polarized plane waves, similarly to simple magnetodielectrics.

In this paper we consider a slab of such composite material with the optical axis orthogonal to the slab surface. For simplicity of the analysis we concentrate on the normal-incidence excitation. In this case both eigenwaves in the slab have the same propagation constant [30]

$$
\beta=k_{0} \sqrt{\varepsilon_{t} \mu_{t}-K^{2}},
$$

where $k_{0}$ is the free-space wavenumber. For the characteristic admittances which are different for the waves traveling in the positive and negative directions of the axis $z$ (denoted below as $Y_{+}$and $Y_{-}$, respectively), the following relation holds [30]:

$$
Y_{ \pm}=Y_{0} \sqrt{\frac{\varepsilon_{t}}{\mu_{t}}}\left(\sqrt{1-\frac{K^{2}}{\varepsilon_{t} \mu_{t}}} \mp j \frac{K}{\sqrt{\varepsilon_{t} \mu_{t}}}\right),
$$

where $Y_{0}=\sqrt{\varepsilon_{0} / \mu_{0}}$ is the free-space wave admittance.

In [32] it was shown that omega composites can be used to realize absorbing layers which are matched to free space. Indeed, one can notice that if the material parameters satisfy the condition

$$
K=\frac{j}{2}\left(\mu_{t}-\varepsilon_{t}\right),
$$

relation (4) gives $Y_{+}=Y_{0}$. Then the reflection coefficient from one side of the slab of an arbitrary thickness $d$ equals $R=0$ and the transmission coefficient reads $T=\exp (-j \beta d)$. Note that the matching condition can be satisfied for arbitrary permittivity and permeability values, provided one can control the coupling coefficient $K$.

If condition (5) is satisfied, the propagation constant of the eigenwaves in the medium reads (3)

$$
\beta=\frac{k_{0}}{2}\left(\varepsilon_{t}+\mu_{t}\right) .
$$

This result shows that the magnetoelectric coupling not only allows one to match the slab to free space, but also makes it easier to realize the backward-wave regime. From formula (6) we see that the refractive index in a matched $\Omega$-slab is negative when

$$
\operatorname{Re}\left\{\varepsilon_{t}+\mu_{t}\right\}<0,
$$

which is easier to satisfy than the usual conditions

$$
\operatorname{Re}\left\{\varepsilon_{t}\right\}<0, \quad \operatorname{Re}\left\{\mu_{t}\right\}<0 .
$$

In particular, it is not necessary to realize negative permeability, which can be more difficult at the optical frequencies than to satisfy (5). Composites with negative permittivity and acceptable losses, on the contrary, are available in optics and can be obtained, for example, using dilute arrays of metal nanoparticles embedded in a dielectric matrix. Like metal split rings [13, 14, 23], metal bianisotropic particles can possess a resonance in the infrared range and, possibly, in the visible. Resonant magnetoelectric coupling of these particles can be sufficient even if the corresponding resonant magnetic polarizability is not high. This expectation is based on the fact that the effect of artificial permeability is of the order of $\mu \sim O\left(k_{0} d\right)^{2}$, while the effect of magneto-electric coupling is an order of magnitude stronger: $K \sim O\left(k_{0} d\right)$. Here $d$ is the characteristic particle size.

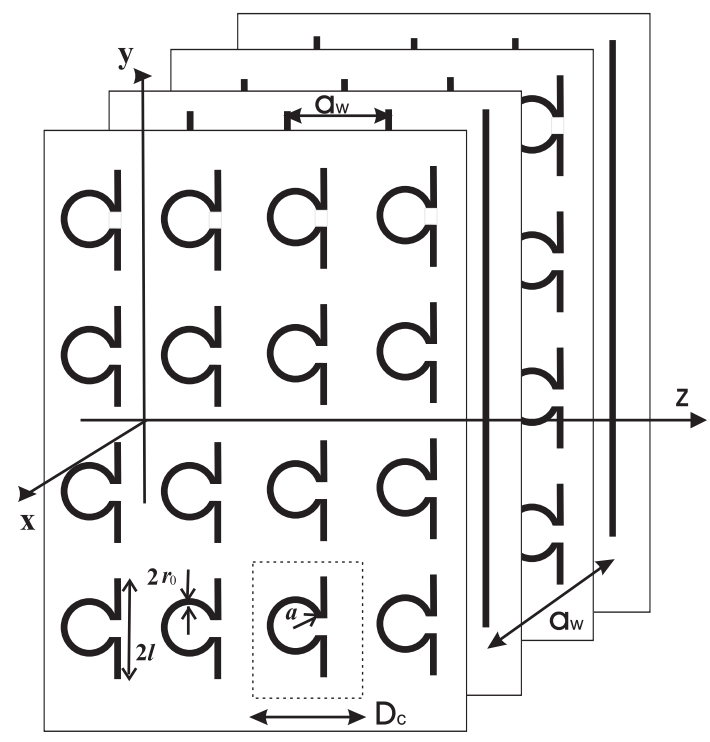

FIG. 1: Geometry of the matched backward-wave structure and of an individual particle. The uniaxial structure has a second identical set of particles lying in the $x-z$ plane (not shown), however that second set does not interact with the eigenwave with the electric field polarized along $y . D_{c}$ is the $\Omega$-lattice unit cell size, $a_{w}$ is the period of the wire lattice.

To study if a realization is possible with a particular realistic dimensions we will next consider an example for the microwave frequency region. Negative permittivity at microwaves can be obtained using a lattice of parallel wires (e.g., [33]). A uniaxial omega composite can be realized as a lattice of pairs of $\Omega$-particles. One particle in the pair is orthogonal to the other $[30,32]$. In this paper we restrict our study by the case of the normal propagation direction. Then, for the simplified structure shown in Fig. 1 all the formulas written above for a uniaxial composite are valid. Our purpose is to show that conditions (5) and (7) correspond to physically achievable geometric parameters of $\Omega$-particles and wires forming the composite medium shown in Fig. 1.

The microwave electromagnetic model of an omega particle represents the particle as a connection of a short wire dipole antenna and a small loop antenna [29]. Small bianisotropic particles can be characterized by dyadic electric and magnetic polarizabilities, which define the relations between induced electric and magnetic dipole 
moments $\mathbf{p}, \mathbf{m}$ and external electric and magnetic fields $\mathbf{E}, \mathbf{H}$ applied to the particle [28]-[30]:

$$
\begin{gathered}
\mathbf{p}=\overline{\bar{\alpha}}_{e e} \cdot \mathbf{E}+\overline{\bar{\alpha}}_{e m} \cdot \mathbf{H} \\
\mathbf{m}=\overline{\bar{\alpha}}_{m e} \cdot \mathbf{E}+\overline{\bar{\alpha}}_{m m} \cdot \mathbf{H}
\end{gathered}
$$

For electrically small particles a lumped-element equivalent circuit models of such antennas give acceptable accuracy [30], and the particle polarizabilities can be expressed in terms of equivalent circuit parameters. If the loop radius $a$ of an $\Omega$-particle shown in Fig. 1 is equal to or larger than the arm length $l$, the polarizability components relevant in the case of the normal propagation can be approximated as

$$
\begin{gathered}
\alpha_{e e}^{y y}=\frac{A}{\omega_{0}^{2}-\omega^{2}+j \omega \Gamma}, \quad A=\frac{l^{2}}{L_{0}}, \\
\alpha_{m m}^{x x}=\frac{B \omega^{2}}{\omega_{0}^{2}-\omega^{2}+j \omega \Gamma}, \quad B=\frac{\pi^{2} \mu_{0}^{2} a^{4}}{L_{0}}, \\
\alpha_{m e}^{x y}=\frac{j \omega D}{\omega_{0}^{2}-\omega^{2}+j \omega \Gamma}, \quad D=\frac{2 \pi^{2} \mu_{0} \varepsilon_{0} \varepsilon_{m} a^{4}}{L_{0} C_{0}},
\end{gathered}
$$

where $\omega_{0}=1 / \sqrt{L_{0} C_{0}}, \Gamma=R_{l} / L_{0}$, and $\varepsilon_{m}$ is the matrix relative permittivity. The matrix is assumed to be non-magnetic: The matrix permeability $\mu_{r}=1$. The equivalent circuit parameters read

$$
\begin{gathered}
R_{l}=\sqrt{\frac{\omega \mu_{0}}{2 \sigma}} \frac{a}{r_{0}}, \quad L_{0}=\mu_{0} a\left[\log \left(\frac{8 a}{r_{0}}\right)-2\right], \\
C_{0}=\frac{\pi l \varepsilon_{0} \varepsilon_{m}}{\log \left(2 l / r_{0}\right)},
\end{gathered}
$$

where $\sigma$ is the metal conductivity, and dimensions $r_{0}$, $a$ and $l$ are defined in Fig. 1. Here we have neglected the electric polarization of the ring compared to that of the dipole portion which is an acceptable approximation for electrically small $\Omega$-particles [30]. However, the bianisotropy of the particle described by formula (13) is practically that of the split ring loaded by a lumped capacitance $C_{0}$. This approximation is suitable for the case $a \geq l$.

For the relative transversal permittivity and permeability and for the magnetoelectric coupling the lowdensity approximation (the particles concentration $N$ is such that the unit cell of the composite is significantly larger than the particles sizes) gives in accordance with [32]:

$$
\varepsilon_{t}=\varepsilon_{r}+\frac{N \alpha_{e e}^{y y}}{\varepsilon_{0}}=\varepsilon_{r}+\frac{N A}{\varepsilon_{0}\left(\omega_{0}^{2}-\omega^{2}+j \omega \Gamma\right)},
$$

$$
\begin{aligned}
& \mu_{t}=\mu_{r}+\frac{N \alpha_{m m}^{x x}}{\mu_{0}}=\mu_{r}+\frac{N B \omega^{2}}{\mu_{0}\left(\omega_{0}^{2}-\omega^{2}+j \omega \Gamma\right)}, \\
& K=N j \frac{\alpha_{m e}^{x y}}{\sqrt{\varepsilon_{0} \mu_{0}}}=-N \frac{\omega D}{\left(\omega_{0}^{2}-\omega^{2}+j \omega \Gamma\right) \sqrt{\varepsilon_{0} \mu_{0}}},
\end{aligned}
$$

where $\varepsilon_{r}$ and $\mu_{r}$ are the relative effective permittivity and permeability of the background material, respectively. In formulas (16)-(18) we use a different notation for the background material permittivity than in (15) because in our structure $\varepsilon_{r} \neq \varepsilon_{m}$. The background permittivity is, of course, affected by the presence of metal wires shown in Fig. 1. However, the capacitance $C_{0}$ is determined by the quasi-stationary electric field in the small spatial domain of the particle. That is why the matrix permittivity $\varepsilon_{m}$ and not $\varepsilon_{r}$ enters $C_{0}$ (see more on this important issue in [34] and [35]).

Conditions (5) and (7) should be satisfied at the same operational frequency $\omega$. Substituting (16)-(18) into (5) we come to equations for the real and imaginary parts of (5):

$$
\begin{gathered}
\frac{2 D}{\sqrt{\varepsilon_{0} \mu_{0}}}=\frac{\left(1-\varepsilon_{r}\right)}{N} \Gamma, \\
\frac{\left(1-\varepsilon_{r}\right)}{N}\left(\omega_{0}^{2}-\omega^{2}\right)=\left(\frac{A}{\varepsilon_{0}}-\frac{B \omega^{2}}{\mu_{0}}\right) .
\end{gathered}
$$

Substituting (16)-(18) into (7) together with (19) and (20) it is possible to show that condition $\varepsilon_{r}<-1$ should hold for simultaneous validity of conditions (5) and (7). This inequality can be satisfied with the negative background permittivity created by the wire medium. If the wave propagates in the plane orthogonal to the wires and the electric field is oriented along them, the wire array behaves as a low-frequency plasma. The permittivity of the wire lattice is then not spatially dispersive, and we have $[33,36]$

$$
\varepsilon_{r}(\omega)=\varepsilon_{m}\left(1-\frac{\omega_{p}^{2}}{\omega^{2}}\right)
$$

In [36] one can find two formulas for the plasma frequency of such wire arrays, the first one,

$$
\omega_{p}^{2}=\frac{2 \pi}{\varepsilon_{0} \mu_{0} a_{w}^{2}\left(\log \frac{a_{w}}{2 \pi r_{w}}+0.5275\right)},
$$

is valid if the radius $r_{w}$ of wires is very small (practically $\left.r_{w}<a_{w} / 100\right)$. If it is not so small $\left(a / 20<r_{w}<a_{w} / 5\right)$, the second formula is applicable:

$$
\omega_{p}^{2}=\frac{2 \pi}{a_{w}^{2} \varepsilon_{0} \mu_{0} \log \frac{a_{w}^{2}}{4 r_{w}\left(a_{w}-r_{w}\right)}} .
$$




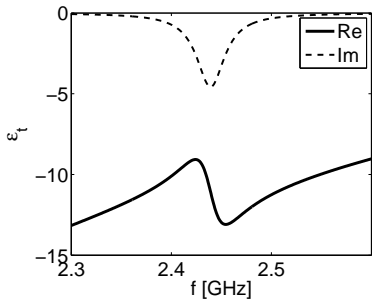

(a)

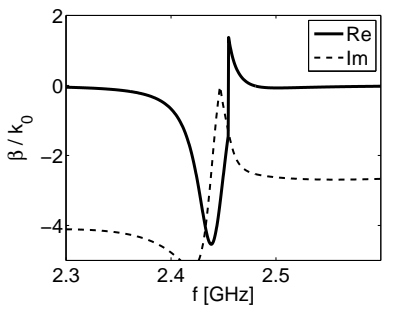

(c)

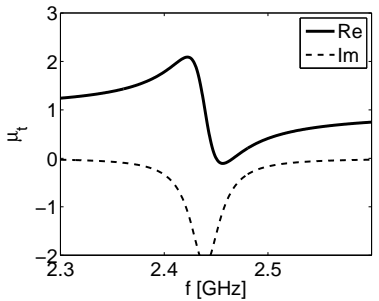

(b)

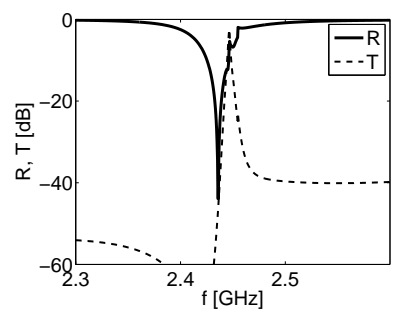

(d)
FIG. 2: Parameters of the bianisotropic layer with the designed lattice geometry. Effective permittivity (a), permeability (b), normalized propagation factor $\beta / k_{0}$ (c), reflection and transmission coefficients in $\mathrm{dB}$ for a $32-\mathrm{mm}$ thick layer (d).

Let us pick up some reasonable values of parameters $\Gamma, \varepsilon_{m}, a, l, r_{0}$ (so that $a>l$ and $a, l \gg r_{0}$ ) and calculate $\omega_{0}$. Then from (19) and (20) we find $\left(1-\varepsilon_{r}\right) / N$ and $\omega$. At the frequency $\omega$ the medium under design is perfectly matched. Then varying $N$ we satisfy the condition $\varepsilon_{r}<$ -1 . Finally we express the needed metal conductivity through $\Gamma$ :

$$
\sigma=\frac{\omega \mu_{0}}{2}\left(\frac{a}{\Gamma L_{0} r_{0}}\right)^{2} .
$$

The results for the particle parameters should satisfy to the following physical conditions:

- Effective medium condition: $D_{c}<\lambda_{\text {eff }} / 2$, where $\lambda_{\text {eff }}=2 \pi / \operatorname{Re}(\beta)$ is the effective wavelength in the medium at the working frequency and $D_{c}=N^{-1 / 3}$ is the size of a unit cell of the $\Omega$-lattice.

- Low concentration condition: $D_{p}=\max (2 a, 2 l)<$ $\min \left(D_{c}, a_{w}\right)$. This means that particles do not touch each other and the wires.

- Metal conductivity: $\sigma$ should be that of a known metal or alloy.

If any of these conditions are violated, one can vary the initial parameters $\Gamma, \varepsilon_{m}, a, l, r_{0}$ and finally find their proper combination. Then we put the wire medium period be equal to the unit cell size $a_{w}=D_{c}$ and find the wire medium plasma frequency and the wire radius $r_{w}$ from formula (21) together with (22) or (23). These formulas allow us to study the frequency properties of the designed structure.
A result obtained for the following design parameters: $\varepsilon_{m}=10, l=3.5 \mathrm{~mm}, a=3.9 \mathrm{~mm}, r_{0}=0.05 \mathrm{~mm}$, is presented in Fig. 2 for the frequency band centered at the particle resonant frequency $f_{0}=2.4393 \mathrm{GHz}$. The perfect matching holds at the frequency $f=2.4359 \mathrm{GHz}$. The parameter $\Gamma=0.02 \cdot \omega_{0}$ corresponds to the conductivity $\sigma \approx 1.6 \cdot 10^{6} \mathrm{1} / \mathrm{Ohm} \cdot \mathrm{m}$. With small metal losses (those of copper) we could not satisfy the effective medium condition. Other parameters were found as explained above: $N=2.34 \cdot 10^{5} 1 / \mathrm{m}^{3}$ that corresponds to $D_{c}=a_{w} \approx 16.23 \mathrm{~mm}$ and $r_{w}=0.07 \mathrm{~mm}$ that allows the backward-wave condition over the particle resonance band. One can see that the permeability is positive in the backward-wave region. The backward-wave regime is due to the bianisotropy. The layer thickness was chosen $d=2 D_{c}$ (the number of particles across the layer is enough to use the effective medium approximation). The thickness resonances happen at much higher frequencies than $f$ (in the region where $\varepsilon_{r}>0$ ).

To conclude, a bianisotropic composite supporting backward waves can be obtained with physically realizable parameters of inclusions. This makes the bianisotropic route to matching a backward-wave composite be prospective for microwave and hopefully for optical metamaterials. Although this matching is perfect for the normal incidence only, the mismatch for oblique incidence should be of the same order as for conventional isotropic slabs with $\mu / \epsilon=\mu_{0} / \epsilon_{0}$. It is also important to notice that the omega slab matching is totally thickness independent and holds even for a semi-infinite lattice.

This work has been supported in part by the Grant Agency of the Czech Republic under project 102/06/1106 "Metamaterials, nanostructures and their applications" and by the Finnish Academy via its Center-of-Excellence program.

[1] V. Shalaev, Nature Photonics 1, 43 (2007)

[2] M. Lapine and S. Tretyakov, IET Microwaves, Antennas \& Propagation, 1, 3 (2007)

[3] V. G. Veselago and E. E. Narimanov, Nature Materials 5, 759 (2006)

[4] Y. Zhang and A. Mascarenhas, Modern Phys. Lett. B 19, $21(2005)$

[5] C. M. Krone, in Encyclopedia on RF and Microwave Engineering, Wiley, Vol. 3, 2303 (2005)

[6] S. A. Ramakrishna, Rep. Prog. Phys. 68, 449 (2005)

[7] A. Lakhtakia, M. W. McCall, and W. S. Weiglhofer, Int. J. Electron. Commun. (AEU) 56, 407 (2002)

[8] J. B. Pendry, Phys. Rev. Lett. 85, 3966 (2000)

[9] J. B. Pendry and S. A. Ramakrishna, J. Phys.: Condens. Matter 15, 6345 (2003)

[10] N. Fang, H. Lee, C. Sun and X. Zhang, Science 308, 534 (2005)

[11] E. Betzig, J. K. Trautman, T. D. Harris, J. S. Weiner and R. L. Kostelak, Science 2511468 (1991) 
[12] R. A. Shelby, D. R. Smith and S. Schultz, Science 292, 77 (2001)

[13] S. Linden, C. Enkrich, M. Wegener, J. Zhou, T. Koschny, C. M. Soukoulis, Science 306, 1351 (2004)

[14] J. Zhou, T. Koschny, M. Kafesaki, E. N. Economou, J. B. Pendry, C. M. Soukoulis, Phys. Rev. Lett. 95, 223902 (2005)

[15] I. V. Lindell, S. A. Tretyakov, K. I. Nikoskinen, and S. Ilvonen, Microwave and Optical Technology Lett. 31, 129 (2001)

[16] P. A. Belov, Microwave and Optical Technology Lett. 37, 259 (2003)

[17] V. A. Podolskiy and E. E. Narimanov, Phys. Rev. B 71, 201101 (2005)

[18] A. Alu, A. Salandrino, N. Engheta, Optics Express 14, 1557 (2006)

[19] A. Pokrovsky and A. Efros, Solid State Communications 129, 643 (2004)

[20] V. A. Podolskiy, A. K. Sarychev, and V. M. Shalaev, Journal of Nonlinear Optical Physics and Materials 11, 65 (2002)

[21] S. Zhang, W. Fan, N. C. Panoiu, K. J. Malloy, R. M. Osgood, S. R. J. Brueck, Phys. Rev. Lett. 95, 137404 (2005).

[22] R. Marques, F. Medina, R. Rafii-El-Idrissi, Phys. Rev. B, 65 (2002) 144440(1-6)

[23] T. Koschny, L. Zhang, and C. M. Soukoulis, Phys. Rev. B 71, 121103(R) (2005)

[24] S.A. Tretyakov, I. Nefedov, A. Sihvola, S. Maslovski and C. Simovski, Journal of Electromagnetic Waves and Applications 17, 695 (2003)
[25] J. B. Pendry, Science 306, 1353 (2004)

[26] S. Tretyakov, A. Sihvola, L. Jylhä, Photonic and Nanostructures, 3107 (2005)

[27] J. Q. Shen, and S. He, J. Phys. A: Math. Gen., 39, 457 (2006)

[28] M. M. Saadoun and N. Engheta, Microwave and Optical Technology Lett. 5, 184 (1992)

[29] C. R. Simovski, A. A. Sochava, T. G. Kharina, B. Sauviac, and S.A. Tretyakov, Journal of Electromagnetic Waves and Applications 11, 1509 (1997)

[30] A. Serdyukov, I. Semchenko, S.A. Tretyakov, A. Sihvola, Electromagnetics of Bi-anisotropic Materials, Theory and Applications (NY, Gordon and Breech Science Publishers, 2001).

[31] T. Kharina, S. Tretyakov, A. Sochava, C. Simovski, S. Bolioli, Electromagnetics, 18423 (1998)

[32] S. Tretyakov and A. Sochava, Electronics Letters, 29, 1048 (1993)

[33] J. B. Pendry, A. J. Holden, W. J. Stewart, and I. Youngs, Phys. Rev. Lett. 76, 4773 (1996)

[34] A. L. Pokrovsky and A. L. Efros, Phys. Rev. Lett. 89, 093901 (2002).

[35] S. A. Tretyakov, I. S. Nefedov, C. R. Simovski, S. I. Maslovski, in: Advances in Electromagnetics of Complex Media and Meta-Materials, S. Zouhdi, A. Sihvola and A. Arsalane, Eds., NATO Series on High Technologies 89, Dordrecht, Kluwer Academic Publishers, 99, 2003

[36] S. A. Tretyakov, Analytical Modeling in Applied Electromagnetics (Norwood, MA, Artech House, 2003) 